\title{
Efectos de la práctica del tai chi chuan sobre la calidad del sueño
}

\author{
Pedro Jesús JIMÉNEZ* \\ Universidad Politécnica de Madrid (España)
}

Recepción: 06/11/2011; Aceptación: 27/02/2012; Publicación: 15/06/2012.

\section{Resumen \\ Effects of Tai Chi Chuan practice on sleep quality}

Objetivo: Este artículo hace un análisis de los estudios internacionales realizados con relación a los efectos del tai chi chuan (también conocido como taichí, taiji o taijiquan) sobre el sueño en sus practicantes con el fin de clarificar el marco de investigación desde el que se está abordando esta problemática, cuáles son sus efectos y las causas que los producen. Método: La búsqueda bibliográfica se realizó sobre siete bases de datos internacionales hasta octubre del año 2011.

Resultados/Conclusiones: Los trabajos de revisión realizados sobre el tema son muy escasos y abordan un escaso número de estudios; mientras que los estudios específicos confirman un tendencia positiva respecto a que el tai chi chuan produce una mejora significativa sobre la estabilidad (interrupción del sueño) y la calidad (eficacia y duración) del sueño nocturno, y una mejora general sobre el sueño diurno, aunque para disfrutar de tales beneficios es necesario un tiempo mínimo de práctica. Las causas fisiológicas que conducen a esta mejora están todavía por determinar.

Palabras clave: Tai chi chuan, trastornos de sueño, calidad de sueño, calidad de vida.

\begin{abstract}
Aim: This paper reviews international studies on the effects of Tai Chi Chuan (a.k.a. taichi, taiji or taijiquan) on sleep with the aim of clarifying the research framework, which are its causes and effects.

Method: The literature search was carried out on the basis of seven international databases on October 2011.

Results/Conclusions: The review works on the subject are scarce and address a small number of studies, while specific works confirm that tai chi chuan produces a significant improvement over the stability (sleep disruption) and quality (efficacy and duration) of nighttime sleep, and an overall improvement on daytime sleep, although to enjoy these benefits its required a minimum of practice. The physiological causes that lead to this improvement are still to be determined.
\end{abstract}

Keywords: Tai Chi Chuan, sleep disorders, sleep quality, quality of life.

\section{Efeitos da prática do tai chi chuan na qualidade do sono}

\section{Resumo}

Objectivo: Este artigo apresenta uma análise dos estudos internacionais realizados relativamente aos efeitos da prática do Tai Chi Chuan (também conhecido por taichi ou taijiquan) no sono dos seus praticantes, com o fim de clarificar o quadro de investigação desta problemática, quais são os seus efeitos e as quais são as causas produzidas.

Método: A análise bibliográfica realizou-se até ao mês de Outubro do ano 2011, tendo por base sete bases de dados internacionais.

Resultados/Conclusões: Os trabalhos de revisão realizados sobre o tema são muito escassos e abordam um limitado número de estudos. Os estudos específicos confirmam uma tendência positiva relativamente à prática do tai chi chuan, produz uma melhoria significativa sobre a estabilidade (interrupção do sono) e qualidade (eficácia e duração) do sono nocturno e uma melhoria geral sobre o sono diurno, mas para desfrutar de tais benefícios é necessário um tempo mínimo de prática. As causas fisiológicas que conduzem a esta melhoria estão, todavia, por determinar.

Palavras-chave: Tai Chi Chuan, transtornos do sono, qualidade do sono, qualidade de vida.

\section{1.- Introducción}

El Tai Chi Chuan (TCC) es una práctica física en forma de coreografía que se caracteriza por realizarse con movimientos lentos, suaves y continuos, fluyendo armoniosamente de una postura a

\footnotetext{
E-mail: pedrojesus.jimenez@upm.es. Dirección: Facultad de Ciencias de la Actividad Física y del Deporte - INEF. Universidad Politécnica de Madrid. C/ Martín Fierro s/n. 28040. Madrid (España).
} 
otra a través del cambio de peso en los miembros inferiores. Históricamente se han desarrollado diferentes escuelas o estilos siendo los más populares los estilos Chen, Yang, Wu y Sun. Cada estilo muestra una personalidad propia que lo diferencia de los demás (Chang et al., 2010; Chen, 2006; Wang, Collet \& Lau, 2004).

El TCC ha sido relacionado con la capacidad de producir numerosos beneficios sobre la salud física y mental de sus practicantes: a nivel cardiorrespiratorio (Brown et al., 1989), sobre la osteoartritis (Brismeé et al., 2007; Ni et al., 2010; Song et al., 2003), el equilibrio (Fong \& Ng, 2006; Gyllensten et al., 2010; Tsang et al., 2004; Wong et al., 2001), el miedo a las caídas (Taggart, 2002; Wolf et al., 1996; Zhang et al., 2006), la depresión, la ansiedad, el ánimo y el estrés (Brown et al., 1995; Galantino et al., 2005; Jin, 1989; Taylor et al., 2006; Toda et al., 2011), la autoestima (Blake y Batson, 2009), la auto-eficacia (Dechamps et al., 2009; Lee et al., 2009; Taylor y Froelicher, 2004), etc. A nivel psicológico, se ha catalogado incluso como "meditación en forma de movimiento" por la capacidad del TCC de sumir a sus practicantes en estados de gran tranquilidad y relajación mental (Taylor et al., 2006; Wang et al., 2010a).

Otro de los beneficios que se asocia a la práctica del TCC, y que va a centrar la temática de este trabajo, es su efecto positivo sobre los trastornos de sueño. El sueño es un proceso psicológico que ha sido estudiado desde hace tiempo por la ciencia, llegando a la conclusión de que no es un fenómeno homogéneo. El sueño se divide en dos fases: la fase REM y la no-REM. La primera se caracteriza por mostrar una alta actividad cerebral acompañada de un movimiento ocular rápido y coordinado, de donde adquiere su nombre; la segunda, se considera de inactividad cerebral y se caracteriza por una reducción progresiva de la tonicidad muscular, con cuatro fases hacia el sueño profundo. Aunque la fisiología del sueño todavía no se comprende totalmente, se acepta que tiene un importante papel en la recuperación de la normalidad en el metabolismo y la actividad neurológica (Valenza, Rodenstein \& Fernández, 2010). Las modificaciones en la estructura, calidad y cantidad de sueño es lo que se ha denominado "disfunción del sueño", y esta alteración puede tener lugar a cualquier edad. Se ha confirmado que las disfunciones del sueño tienen importantes efectos sobre las funciones cerebrales y dan lugar a diferentes alteraciones psicológicas (dificultad de concentración y pérdida de memoria), conductuales (estrés emocional, depresión, fatiga, irritabilidad) y fisiológicas (aumento en los niveles de cortisol, incremento de la actividad simpática, y la presión arterial e incremento del dolor) (Valenza, Rodenstein \& Fernández, 2010).

Los estudios sobre la influencia del TCC en los trastornos de sueño se ubican dentro del campo de la psicología, y según la jerarquía establecida por Spidurso, Francis y MacRae (2005) en el ámbito de la calidad de vida, y en particular, en el marco del bienestar corporal. En las revisiones sistemáticas específicas encontradas sobre los efectos psicológicos que aporta la práctica del TCC sobre los trastornos del sueño, sólo tres estudios (Wang et al., 2009; Wang et al., 2010a y Zhang, Layne, Lowder \& Liu, 2011) hacen alusión al sueño en su análisis y señalan efectos positivos por la práctica de esta actividad, si bien la problemática es abordada desde un punto de vista meramente descriptivo y resumido, sin entrar a desarrollar una reflexión específica sobre la importancia de los resultados. El resto de revisiones sistemáticas encontradas no abordaron este aspecto (Chang et al., 2010; Dechamps, Lafont \& Bourdel-Marchasson, 2007; Hall et al., 2009; Lee, Pittler \& Ernst, 2008; Rogers, Larkey \& Keller, 2009; Wang et al., 2010a; Wang, Collet \& Lau, 2004).

En las revisiones genéricas sobre los efectos del TCC en la salud (Field, 2010; Kuramoto, 2006; Li et al., 2011) y en las que incluyen los efectos del TCC sobre el sueño pero dentro de los términos medicina complementaria y alternativa (Cherniack, 2006; Sarris \& Byrne, 2011), terapias mente y cuerpo (Innes, Selfe \& Vishnu, 2010; Kemper et al., 2011; Kozasa et al., 2010), meditación en movimiento (Rogers, Keller \& Larkey, 2010), terapias energéticas (Cuellar, Rogers \& Hisghman, 2007) o sueño en general (Stein \& Pu, 2011; Valenzuela, Rodenstein \& Fernández, 2010), el problema se trata también de forma tangencial y resumida, realizando simples descripciones sobre los estudios localizados y se analiza, en la mayor parte de los casos, un número muy reducido de estudios. Sin embargo, pueden destacarse las revisiones de Kemper et al. (2011) y Rogers, Keller y Larkey (2010), en las que se aborda esta problemática desde una perspectiva innovadora. Ambos estudios evalúan cuáles son los beneficios psico-sociales percibidos o esperados por los practicantes y los motivos que llevan a las personas a iniciarse en estas prácticas, y afirman que la expectativa de conseguir "dormir mejor" es un motivo importante para iniciarse en las mismas. 
Dada la escasa atención que ha recibido el estudio de los efectos de la práctica del TCC sobre los trastornos de sueño en las revisiones sistemáticas y generales encontradas en la literatura científica, el objetivo de este trabajo es analizar los estudios específicos que se han realizado sobre los efectos del TCC sobre el sueño con el fin de clarificar el marco de investigación desde el que se está analizando esta problemática y cuáles son los beneficios que produce.

\section{2.- Método}

Para localizar los estudios se utilizaron siete bases de datos electrónicas (Medline-Pubmed, Sircus, Pascal, ScienceDirect, SportDiscuss, Science Citation Index y BIOSIS) y se eligieron como palabras clave, teniendo en cuenta el trabajo de Pérez-Gutiérrez, Gutiérrez-García y Escobar Molina (2011), diferentes combinaciones de los términos: Tai Chi, T’ai Chi, Tai Ji, complementary medicine, alternative medicine, mind-body methods, meditative movement, psychology, sleep disorders, insomnia, y quality of life, y sus correspondientes términos en castellano.

La revisión se realizó hasta octubre del año 2011. Los criterios de inclusión fueron: a) que los estudios tuviesen como objetivo de medición los efectos del TCC sobre los trastornos de sueño, b) que estuviesen escritos en inglés o en castellano y c) que fuesen investigaciones originales, aunque también se consultaron revisiones sobre la temática para la localización de trabajos. No se incluyeron actas de congresos, resúmenes, pósters, o artículos repetidos.

\section{3.- Resultados}

El número de estudios encontrados que cumplieron los criterios de inclusión y a los que se pudo tener acceso fue de doce. Siete fueron estudios experimentales con grupos de control (EE) y cinco no experimentales (NE). Los trabajos se realizaron en Estados Unidos, Irán (Hosseini et al., 2011) y Tailandia (Taboonpong et al., 2008) entre los años 2001-2011, y los estilos de TCC utilizados fueron el Yang en sus versiones simplificadas de 5, 8, 10, 13, 20 y 24 movimientos; Chen, sin especificar sus movimientos; e incluso uno creado a propósito para la investigación (Tai Chi Easy). La duración de las intervenciones osciló entre 5-25 semanas y la frecuencia de clases fue de 1-3 veces por semana con una duración de 20 minutos a 2 horas por sesión. El número de participantes osciló entre 8 y 349 personas, con una edad superior a los 50 años en 8 estudios, y 3 estudios se realizaron con adolescentes. Todos los estudios encontrados confirmaron una mejora significativa en la calidad del sueño nocturno y diurno por la práctica del TCC, si bien el sueño diurno sólo fue evaluado en dos intervenciones (Tabla 1).

De las doce intervenciones analizadas, cuatro de ellas (Hernández-Reif, Field \& Thimas, 2001; Hoffmann-Smith et al., 2009; Jahnke et al., 2010; Wall, 2005) abordaron el problema de forma secundaria como un parámetro más dentro de su objetivo de investigación y sin utilizar un instrumento de evaluación específico para el sueño; cinco (Caldwell et al. 2009; Hosseini et al., 2011; Lavretsky et al., 2011; Taboonpong et al., 2008; Wang et al., 2010), aunque sí utilizaron un instrumento específico de evaluación, abordan la evaluación de forma general sin describir sobre qué aspectos o parámetros del sueño se produjo la mejora; y tres (Irwin et al., 2008; Li et al., 2004; Yeh et al., 2008) analizaron los efectos del TCC sobre los trastornos de sueño en profundidad, con instrumentos específicos y diferenciando los aspectos de mejora.

a) Estudios que no utilizan instrumentos de medición específicos para el sueño. Tanto el estudio de Hoffmann-Smith et al. (2009), centrado en los efectos del TCC sobre personas adultas con ansiedad moderada, como el de Hernández-Reif, Field y Thimas (2001), que analizaba los beneficios de esta práctica en jóvenes con síndrome de ADHD, confirmaron, con intervenciones de tan sólo 10 y 5 semanas respectivamente, efectos positivos del TCC sobre el sueño; sin embargo, su medición se realizó de manera secundaria como un parámetro más dentro de otros instrumentos: el Hamilton-Anxiety Psychiatric Scale en el primero, y el Conners Teacher Rating Scale Revised en el segundo. Los estudios de Jahnke, Larkey y Rogers (2010) y Wall (2005) no utilizaron ningún instrumento de medición específico ni complementario para este parámetro, si bien el primero al menos, incluyó una pregunta de cuestionario: ¿cuándo has experimentado mala calidad de sueño en el mes pasado? $0=$ Nunca, $1=$ casi nunca, $2=$ algunas veces, $3=$ a menudo, $4=$ muy a menudo. 
Tabla 1

Resumen de los estudios que analizan los efectos del TCC sobre el sueño

\begin{tabular}{|c|c|c|c|c|c|c|c|c|c|c|}
\hline Estudios & $\mathbf{n}$ & Género & Edad & $\begin{array}{c}\text { Estilo TCC } \\
\text { № mov } \\
\end{array}$ & $\begin{array}{c}\text { Duración } \\
\text { (semanas) }\end{array}$ & Frecuencia & $\begin{array}{c}\text { Instrumento } \\
\text { evaluación }\end{array}$ & Mejora sueño nocturno & $\begin{array}{c}\text { Mejora } \\
\text { sueño diurno } \\
\end{array}$ & Patología \\
\hline \multicolumn{11}{|l|}{ NE } \\
\hline $\begin{array}{l}\text { Hernández } \\
\text { et al. (2001) }\end{array}$ & 13 & $\begin{array}{l}11 \mathrm{v} \\
2 \mathrm{~m}\end{array}$ & 14.5 & $\begin{array}{l}\text { SE } \\
\text { SE }\end{array}$ & 5 & $\begin{array}{c}2 \mathrm{v} / \mathrm{s} \\
30 \mathrm{~min} / \mathrm{s}\end{array}$ & CTRS & -- & $\mathrm{SI}(\mathrm{p}=0.000)$ & ADHD \\
\hline $\begin{array}{l}\text { Hoffmann et } \\
\text { al. (2009) }\end{array}$ & 19 & $\begin{array}{c}5 \mathrm{v} \\
14 \mathrm{~m}\end{array}$ & 52 & $\begin{array}{l}\text { Yang } \\
24\end{array}$ & 10 & $\begin{array}{l}2 \mathrm{v} / \mathrm{s} \\
1 \mathrm{~h} / \mathrm{s}\end{array}$ & HAM-A & $\mathrm{SI}(\mathrm{p}=\mathrm{SE})$ & - & $\begin{array}{l}\text { Ansiedad } \\
\text { moderada }\end{array}$ \\
\hline Jahnke et al. & 349 & $\begin{array}{c}53 \mathrm{v} \\
296 \mathrm{~m}\end{array}$ & 73 & $\begin{array}{l}\mathrm{SE} \\
\mathrm{SE}\end{array}$ & $8-10$ & $\begin{array}{l}1-2 \mathrm{v} / \mathrm{s} \\
1 \mathrm{~h} / \mathrm{s}\end{array}$ & 1item en PSS & SI $(p=0.42)$ & - & Sujetos sanos \\
\hline $\begin{array}{l}\text { Taboonpong } \\
\text { et al. (2008) }\end{array}$ & 25 & $\begin{array}{l}10 \mathrm{v} \\
15 \mathrm{~m}\end{array}$ & $60-80$ & $\begin{array}{l}\mathrm{SE} \\
18 \\
\end{array}$ & 12 & $\begin{array}{c}3 \mathrm{v} / \mathrm{s} \\
22 \mathrm{~min} / \mathrm{s}\end{array}$ & PQSI & SI $(p<0.01)$ & - & Sujetos sanos \\
\hline Wall (2005) & 11 & $\begin{array}{l}5 \mathrm{v} \\
6 \mathrm{~m}\end{array}$ & $11-13$ & $\begin{array}{c}\text { Yang } \\
13\end{array}$ & 5 & $\begin{array}{l}1 \mathrm{v} / \mathrm{s} \\
1 \mathrm{~h} / \mathrm{s}\end{array}$ & $\begin{array}{c}1 \text { item en } \\
\text { cuestionario }\end{array}$ & $p=S E$ & - & Sujetos sanos \\
\hline $\begin{array}{l}\text { EE } \\
\text { Caldwell et } \\
\text { al. }(2009)\end{array}$ & 29 & $\begin{array}{c}4 \\
24 \mathrm{~m} \\
-\end{array}$ & $\begin{array}{c}21.7 \\
( \pm 2.2)\end{array}$ & $\begin{array}{l}\text { Chen } \\
\text { SE }\end{array}$ & 15 & $\begin{array}{c}2 \mathrm{v} / \mathrm{s} \\
50 \mathrm{~min} / \mathrm{s}\end{array}$ & PQSI & $\mathrm{SI}(\mathrm{p}=\mathrm{SE})$ & - & Sujetos sanos \\
\hline $\begin{array}{l}\text { Hosseini et } \\
\text { al. }(2011)\end{array}$ & 27 & $14 \mathrm{v}$ & $\begin{array}{r}68.74 \\
-( \pm 5.4) \\
\end{array}$ & $\begin{array}{l}\mathrm{SE} \\
10\end{array}$ & 12 & $\begin{array}{c}3 \mathrm{v} / \mathrm{s} \\
20-25 \mathrm{~m} / \mathrm{s}\end{array}$ & PQSI & SI $(p=0.031)$ & - & Sujetos sanos \\
\hline $\begin{array}{l}\text { Irwin et al. } \\
\text { (2008) }\end{array}$ & 59 & $\begin{array}{l}18 \mathrm{v} \\
41 \mathrm{~m}\end{array}$ & $\begin{array}{c}69.8 \\
( \pm 6.2)\end{array}$ & $\begin{array}{c}\text { Yang } \\
20\end{array}$ & 25 & $\begin{array}{c}3 \mathrm{v} / \mathrm{s} \\
40 \mathrm{~min} / \mathrm{s}\end{array}$ & PQSI & $\begin{array}{l}\text { SI }(\mathrm{p}=0.05) \text {. Calidad de sueño }(\mathrm{p}< \\
0.05) \text {; duración }(\mathrm{p}<0.01) \text {; eficacia }(\mathrm{p}< \\
0.05) \text {; trastornos del sueño }(\mathrm{p}<0.01)\end{array}$ & - & $\begin{array}{l}\text { Problemas de } \\
\text { sueño }\end{array}$ \\
\hline $\begin{array}{l}\text { Lavretsky et } \\
\text { al.(2011) }\end{array}$ & 33 & $10 \mathrm{v}$ & $\begin{array}{c}69.1 \\
( \pm 7.0)\end{array}$ & $\begin{array}{l}S E \\
20\end{array}$ & 10 & $\begin{array}{l}1 \mathrm{v} / \mathrm{s} \\
2 \mathrm{~h} / \mathrm{s}\end{array}$ & PQSI & SI $(p=0.003)$ & - & Depresión \\
\hline $\begin{array}{l}\text { Li et al. } \\
(2004)\end{array}$ & 62 & $\begin{array}{l}10 v \\
52 \mathrm{~m}\end{array}$ & $\begin{array}{c}75.3 \\
( \pm 7.8)\end{array}$ & $\begin{array}{c}\text { Yang } \\
8\end{array}$ & 24 & $\begin{array}{l}3 \mathrm{v} / \mathrm{s} \\
1 \mathrm{~h} / \mathrm{s}\end{array}$ & $\begin{array}{c}\text { PQSI } \\
\text { ESS }\end{array}$ & $\begin{array}{l}\text { SI }(p=0.001) \\
\text { (Calidad de sueño }(p<0.01) ; \text { duración } \\
(p<0.01) ; \text { eficacia }(p<0.01) ; \text { tiempo } \\
\text { empleado en dormir }(p<0.01) ; \\
\text { trastornos del sueño }(p<0.01)\end{array}$ & $\operatorname{SI}(p=0.002)$ & $\begin{array}{l}\text { Problemas de } \\
\text { sueño }\end{array}$ \\
\hline $\begin{array}{l}\text { Yeh et al. } \\
(2008) .\end{array}$ & 8 & $\begin{array}{l}4 \mathrm{v} \\
4 \mathrm{~m}\end{array}$ & $\begin{array}{c}64.2 \\
( \pm 16.2) \\
-\end{array}$ & $\begin{array}{c}\text { Yang } \\
5\end{array}$ & 12 & $\begin{array}{l}2 \mathrm{v} / \mathrm{s} \\
1 \mathrm{~h} / \mathrm{s}\end{array}$ & ECG-B S M & $\begin{array}{c}\text { SI }(p=0.04) \\
\text { Sueño estable }(p=0.04) \\
\text { Sueño instable }(p<0.01)\end{array}$ & - & $\begin{array}{c}\text { Fallo } \\
\text { cardiaco }\end{array}$ \\
\hline $\begin{array}{l}\text { Wang et al. } \\
\text { (2010) }\end{array}$ & 33 & $\begin{array}{c}5 \mathrm{v} \\
28 \mathrm{~m}\end{array}$ & $\begin{array}{c}49.7 \\
( \pm 11.8)\end{array}$ & $\begin{array}{c}\text { Yang } \\
10\end{array}$ & 12 & $\begin{array}{l}2 \mathrm{v} / \mathrm{s} \\
1 \mathrm{~h} / \mathrm{s}\end{array}$ & PQSI & SI $(p=0.01)$ & - & Fibromialgia \\
\hline
\end{tabular}

Nota: $\mathrm{NE}=$ No experimental; $\mathrm{EE}=$ Experimental; $\mathrm{v}=$ varones, $\mathrm{m}=$ mujeres; $\mathrm{v} / \mathrm{s}=$ veces por semana; $\mathrm{h} / \mathrm{s}=$ horas por sesión; $\mathrm{min} / \mathrm{s}=$ minutos por sesión; $\mathrm{SE}=\mathrm{Sin}$ especificar. Nota: PQSI= Pittsburgh Sleep Quality Index; CTRS= Conners Teacher Rating Scale Revised; HAM-A= Hamilton-Anxiety Psychiatric Scale; ESS= Epworth Sleepiness Scale; ECG-B SM= ECG-based Spectogram Method; SI= Mejora significativa; ADHD= Attention Deficit Hyperactivity Disorder. 
b) Estudios que utilizan instrumentos específicos de medición pero abordan la problemática de forma genérica. Dentro de este apartado se incluyen las intervenciones de Caldwell et al. (2009), enfocada a jóvenes universitarios estadounidenses y que analizó los efectos del TCC sobre el sueño, la auto-eficacia, el ánimo, la fuerza y el equilibrio; Hosseini et al. (2011), que intervino específicamente para analizar el sueño en centros de tercera edad en Irán; Lavretsky et al. (2011), que comparó los efectos de la medicación en ancianos sobre el sueño, la depresión, la ansiedad, la cognición, la resilencia y la calidad de vida; Taboonpon et al. (2008) que intervinieron con ancianos tailandeses analizando las mejoras sobre la calidad del sueño, el bienestar, la fuerza y la flexibilidad; y Wang et al. (2010), que analizó los efectos del TCC en pacientes norteamericanos con fibromialgia evaluando las mejoras sobre los síntomas de esta patología, la depresión, el dolor y el sueño. Todos estos trabajos incluyeron como instrumento de medición específico el Pittsburg Sleep Quality Index (PQSI), que evalúa el sueño nocturno en las dimensiones de latencia (el tiempo que se tarda en dormir), duración (el número de horas de sueño), eficacia (la relación entre el tiempo que uno está en la cama y el tiempo que duerme), trastornos de sueño (levantarse en medio de la noche), utilización de medicación para el sueño y disfunción diurna (tener dificultades para mantenerse despierto durante el día). Sin embargo, y aunque los autores señalan una mejora significativa en la calidad del sueño de los practicantes, ninguno indica en qué dimensión/es del test se registró dicha mejora.

c) Estudios que analizan en profundidad los efectos del TCC sobre el sueño. Los estudios experimentales de Li et al. (2004), Irwin, Olmstead y Motivala (2008) y Yeh et al. (2009) destacan por su gran calidad de diseño de investigación y, en los dos primeros, porque además se realizaron en centros médicos especializados en el sueño.

El trabajo de Li et al. (2004) utilizó como instrumentos de medición tanto el PSQI, para evaluar el sueño nocturno, como el Epworth Sleepiness Scale (ESS), para evaluar el sueño diurno o la tendencia a dormirse durante el día; el de Irwin, Olmstead y Motivala (2008) se apoyó únicamente en el PQSI; y el de Yeh et al. (2009), considerando que en el sueño influyen diferentes variables fisiológicas (respiración, presión sanguínea, activación simpática, etc.) que no se recogen en los instrumentos de medición utilizados tradicionalmente (PSQI, ESS, encefalograma), diseñó un método de evaluación específico para su investigación basado en una prueba espectográfica en la que se combina el análisis de los patrones del sueño con la valoración de las alteraciones en el ritmo cardíaco y respiratorio.

Li et al. (2004) encontraron que 24 semanas de práctica de TCC, un hora por sesión tres veces por semana, produjeron una mejora significativa en los parámetros de calidad y trastornos del sueño, duración, eficacia y tiempo que se tarda en dormir, en el sueño nocturno, y una mejora general en el sueño diurno (ver Tabla 1), en ancianos diagnosticados con problemas moderados de sueño, en comparación a los que participaban en programa de ejercicio físico moderado. En particular los practicantes de TCC tardaron 18 minutos menos respecto al tiempo para dormirse, y ganaron 48 minutos más de sueño nocturno.

Irwin, Olmstead y Motivala (2008) también encontraron que 25 semanas de práctica, 3 veces por semana y 40 minutos por sesión, también produjeron mejoras significativas en los parámetros de calidad y trastornos del sueño, eficiencia y duración (ver Tabla 1), en el sueño nocturno en personas mayores diagnosticadas con problemas moderados de sueño, pero que 16 semanas de práctica, con la misma dosificación, eran insuficientes, confirmando que un mínimo de tiempo de práctica es necesario para recoger los beneficios del TCC.

Además, hay que destacar que estos autores diferenciaron en sus muestras, antes de comenzar su intervención, entre aquellos participantes que tenían mejor o peor calidad de sueño según habían puntuado en el PSQI por debajo, por encima, o igual a 5, confirmando que estas mejoras fueron más significativas en aquellos pacientes que partían con una mala calidad de sueño. Aún así, los autores también señalan que falta por investigar los efectos de esta práctica sobre pacientes con problemas crónicos de insomnio.

Un año más tarde, Yeh et al. (2009) realizaron otro estudio experimental de gran calidad para analizar los beneficios del TCC en la estabilidad del sueño sobre pacientes con antecedentes de fallo cardíaco y disfunción sistólica ventricular izquierda. Los problemas de insomnio y 
fragmentación del sueño son comunes en pacientes con problemas cardíacos y afectan no solo a la eficiencia del sueño y la reducción del tiempo total durmiendo, sino que también reducen la calidad de vida de estos pacientes. Mejorar el sueño y el proceso respiratorio en estos pacientes es así muy importante.

Aunque el tamaño muestral en la investigación fue reducido, el estudio encontró que 12 semanas de práctica de TCC, dos veces por semana y una hora por sesión, eran suficientes para confirmar una mejora significativa en la estabilidad del sueño de estos pacientes, mayor estabilidad en el ritmo respiratorio y la fisiología cardiaca (mejoras en la tensión arterial y disminución en las arritmias), y confirmó una correlación positiva en la calidad de vida de los participantes. Para estos autores, el TCC no sólo es una opción no farmacológica para mejorar el sueño en las personas, sino en especial para los pacientes con antecedentes cardíacos.

Respecto a las causas que producen estos efectos positivos, pero que todavía quedan por investigar, Irwin, Olmstead y Motivala (2008), Li et al. (2004) y Yeh et al. (2008), señalan como posibles responsables a la relajación y el trabajo respiratorio que acompaña al TCC y a sus efectos sobre el sistema nervioso simpático, cuya activación se ha asociado a los problemas crónicos de insomnio en ancianos, perfil de la muestra con la que han realizado sus trabajos. Sin embargo, no se debe descartar la influencia del efecto placebo como han expuesto algunos estudios sobre los efectos psicológicos del TCC y que pueden ser debidas a las expectativas de los participantes en las que se asocia esta actividad con la salud y la tranquilidad mental, dando lugar al síndrome de profecía auto cumplida por sugestión (Brown et al., 1995; Jin, 1989, 1992; Yau \& Packer, 2002).

\section{4.- Discusión}

La escasa información específica que se ha encontrado en los estudios de revisión generales y específicos sobre los efectos del TCC sobre el sueño tiene su razón en el escaso número de estudios realizados sobre esta temática. No obstante, se puede señalar que si bien estos trabajos enfocan sus revisiones bajo el formato de descripción general de los efectos que genera la práctica de esta actividad o sobre el análisis exclusivo de su diseño experimental, estamos de acuerdo con Li et al. (2011) cuando señalan que en los estudios de revisión sería necesario introducir ítems específicos en relación a los condicionantes propios que afectan a la implementación del TCC (lugar de práctica, experiencia de los instructores, diseño de la sesión, metodología de enseñanza, adaptaciones de los ejercicios en función de las necesidades de los practicantes etc.) con el fin de poder valor con mayor profundidad los resultados en los estudios.

Respecto a los estudios específicos de carácter experimental y no experimental analizados, aunque todos los trabajos coinciden en afirmar que la práctica del TCC tiene efectos positivos sobre la calidad del sueño nocturno y diurno, estos resultados deben ser considerados con cierta precaución. En primer lugar, porque la mayor parte de los estos estudios han abordado el problema de forma secundaria, y en segundo lugar, porque el diseño de investigación en muchos trabajos es de baja calidad (muestras reducidas, carencias en los criterios de inclusión y exclusión de los participantes, carencias en la estandarización de la medición de los resultados, etc.). Este último aspecto hace tiempo que viene siendo señalado en la bibliografía por diferentes autores que hacen estudios de revisión sistemática sobre los efectos del TCC en diferentes ámbitos de la salud y que recomiendan a los autores que adopten en el diseño de sus intervenciones los criterios establecidos en los diferentes instrumentos de evaluación utilizados (Consolidated Standards for Reporting Trials (CONSORT); Jadad Score, PEDro rating scale, National Institute for Health and Clinical Excellence (NICE) etc.) (Hall et al. 2009; Li et al., 2011; Low et al., 2009).

Con relación al tiempo mínimo de práctica necesario para disfrutar de mejoras en la calidad de sueño por la práctica de TCC, el interrogante no está resuelto. Aunque el estudio de HernándezReif, Field y Thimas (2001) parece confirmar que con sólo cinco semanas de práctica, dos veces en semana en sesiones de 30 minutos, son suficientes e incluso que son capaces de mantenerse dichas mejoras incluso en las dos semanas posteriores a la intervención, los trabajos más especializados de Irwin, Olmstead y Motivala (2008) y Yeh et al. (2008), indican que esta cuestión no es tan sencilla. Si para Yeh et al. (2008) doce semanas de práctica, dos veces en semana y una hora de duración eran suficientes para confirmar una mejora significativa en la estabilidad del sueño en 
pacientes con fallo cardíaco, mejorar la estabilidad en el ritmo respiratorio y la fisiología cardiaca (mejoras en la tensión arterial y disminución en las arritmias), Irwin, Olmstead y Motivala (2008) registraron que 16 semanas de práctica no producían ningún registro positivo en la calidad de sueño. La heterogeneidad en los protocolos de medición, los instrumentos utilizados, los parámetros analizados e incluso otros factores relativos a la propia implementación del TCC pueden ser la causa para no llegar todavía a un acuerdo.

En cuanto a los estilos de TCC utilizados en las intervenciones se puede destacar que el estilo Yang es el más utilizado, e incluso que dos estudios adoptan formas específicamente "inventadas" para la investigación. Que el estilo Yang sea el más utilizado en las intervenciones coincide con los resultados encontrados en otros estudios de revisión realizados sobre los efectos del TCC a nivel psicológico (Dechamps, Lafont \& Bourdel-Marchasson, 2007; Jiménez, Meléndez y Albers, 2012; Taylor et al., 2006; Wang et al., 2010a) y tiene sus causas en que es el estilo más practicado a nivel mundial (Low et al., 2009), y el que mejor se adecua a las personas mayores (Dechamps, Lafont \& Bourdel-Marchasson, 2007), población sobre la que se centran la mayor parte de los estudios.

Resulta interesante confirmar que no solo las formas simplificadas del estilo Yang, sino también las formas "inventadas" a propósito en algunas investigaciones, son capaces de proporcionar efectos positivos sobre el sueño de sus practicantes, porque abre el interrogante sobre si dichos beneficios están en la misma proporción a los que aportan las formas tradicionales. Por ejemplo, en este estudio se ha comprobado que una forma simplificada del estilo Yang con tan sólo cinco movimientos es capaz de producir efectos positivos sobre el sueño. Otros estudios, donde se han utilizado formas de TCC "inventadas" a propósito para afrontar diferentes patologías específicas de pacientes, también han registrado efectos positivos en sus resultados, tal es el caso del "Tai Chi: Moving for Better Balance" (ocho movimientos) para problemas de equilibrio (Li et al. 2009), el Tai Chi Yang style exercise program (STEP) (Chen et al., 2006) o el Tai Chi Qigong (TCQ) (Chen et al., 2008) creado para pacientes con problemas de osteoatrosis (Chen et al. 2006; Rogers, Larkey \& Keller, 2009; Wang, Collet \& Lau, 2004).

Las mejoras en la calidad de sueño registradas por la práctica del TCC en pacientes con patologías específicas como fallo cardíaco (Yeh et al., 2008), síndrome de déficit de atención con hiperactividad (ADHD) (Hernández-Reif et al., 2001), fibromialgia (Wang et al., 2010), ansiedad moderada, estado de ánimo y depresión (Cadwell et al., 2009; Hoffmann-Smith et al., 2009; Lavretsky et al., 2011) son muy interesantes porque, aunque el número de estudios es todavía muy reducido, abren un línea de investigación atractiva sobre los posibles efectos sumativos o multiplicativos sobre la salud que tiene la práctica de esta actividad. Además, y tomando como referencia el estudio de Valenza, Rodenstein y Fernández (2010), también sería interesante ampliar los estudios al análisis de los efectos que tiene el TCC sobre problemas específicos asociados con las disfunciones del sueño y que afectan a la conducta (irritabilidad, ansiedad, depresión o falta de ánimo, trastornos obsesivos, esquizofrenia), cognición (problemas de memoria, concentración, aprendizaje), alteraciones hemodinámicas (incremento del ritmo cardíaco, presión sanguínea actividad simpática), patologías coronarias, el dolor, la fatiga y las alteraciones en la función músculo-esquelética.

Por último, señalar que algunos estudios (Li et al. 2004; Yeh et al., 2008; Spirduso, Francis \& MacRae, 2005) también muestran cómo otras fórmulas de actividad físico-deportiva también son capaces de producir efectos positivos sobre la calidad del sueño de sus practicantes y que también faltaría por especificar cuáles son las diferencias específicas que se producen entre el TCC y esas prácticas físico-deportivas con relación a los diferentes parámetros y disfunciones que afectan al sueño.

\section{5.- Conclusiones}

Aunque el número de estudios que analizan los efectos del TCC sobre el sueño todavía es reducido y predominan los estudios de baja calidad en el diseño experimental, aunque con importantes excepciones, las investigaciones realizadas confirman una tendencia positiva respecto a que el TCC aporta beneficios en sus practicantes en la estabilidad (interrupción del sueño) y la 
calidad (eficacia, duración y tiempo que se tarda en dormirse) del sueño nocturno y una mejora general sobre el sueño diurno.

Estos efectos positivos tienden a ser más evidentes en las personas que parten de una mala calidad de sueño, y parecer ser que para disfrutar de los beneficios de esta actividad es necesario un tiempo mínimo de práctica, aunque todavía no es evidente el número de semanas y la dosificación: días por semana y tiempo por sesión.

Las mejoras en la calidad de sueño registradas por la práctica del TCC en pacientes con patologías cardíacas, depresión, ansiedad o hiperactividad son muy interesantes ya que abren un línea de investigación atractiva sobre los posibles efectos sumativos o multiplicativos sobre la salud que tiene la práctica de esta actividad.

Es necesario realizar un mayor número de estudios específicos a nivel fisiológico para determinar las posibles causas que producen las mejoras registradas; ampliar el interés hacia el análisis de los efectos de esta actividad sobre aquellas patologías asociadas a la disfunción del sueño; y confirmar cuáles son las diferencias y sobre que parámetros específicos del sueño tiene influencia cada estilo y forma de TCC y su comparativa respecto a otras actividades físicodeportivas.

\section{Referencias}

Blake, H., \& Batson, M. (2009). Exercise intervention in brain injury: a pilot randomized study of Tai Chi Qigong. Clinincal Rehabilitation, 23, 589-598.

Brismée, J.M., Piage, R., Chyu, M.C., Boatright, J.D., Hagar, J.M., et al. (2007) Group and home-based tai chi in elderly subjects with knee osteoarthritis: a randomized controlled trial, Clinical Rehabilitation, 21 (2), 99-111.

Brown, D., Wang, Y., Ward, A., Ebbeling, C.B., Cara, B., et al. (1995). Chronic psychological effects of exercise and exercise plus cognitive strategies, Medicine and Science in Sports and Exercise, 27(5), 765-775.

Brown, D., Mucci, W., Hetzler, R., \& Knowlton, R. (1989). Cardiovascular and Ventilatoriy Responses During Formalized T'ai Chi Chuan Exercise, Research Quarterly for Exercise and Sport, 60(3), 246-250.

Caldwell, K., Harrison, M., Adams, M., \& Triplett, N.T. (2009). Effects of Pilates and Taiji Quan training on self-efficacy, sleep quality, mood, and physical performance, of college students. Journal of Bodywork and Movement Therapies, 13, 155-163.

Chang, Y.K., Nien, Y.H., Tsai, CL., \& Etnier, J. (2010). Physical Activity and Cognition in Older Adults: The Potential of Tai Chi Chuan, Journal of Aging and Physical Activity, 18, 451-472.

Chen, K.M., Chen, W.T., \& Huang, M.F. (2006). Development of the simplified Tai Chi exercise program (STEP) for frail older adults. Complementary Therapies in Medicine, 14, 200-206.

Chen, C.H., Yen, M., Fetzeer, S., Lo, L.H., \& Lan, P. (2008). The Effects of Tai Chi Exercise on Elders with Osteoarthritis: A Longitudinal Study, Asian Nursing Research, 2(4), 235-241

Cherniack, E.P. (2006). The use of alternative medicine for the treatment of insomnia in the elderly, Psychogeriatrics, 6, 21-30.

Cuellar, N.G., Rogers, A.E., \& Hisghman, V. (2007). Evidenced Based Research of Complementary and Alternative Medicine (CAM) for Sleep in the Community Dwelling Older Adult, Geriatric Nursing, 28(1), 46-52.

Dechamps, A., Lafont, L., \& Bourdel-Marchasson, I. (2007). Effects of Tai Chi exercises on selfefficacy and psychological health, European Review of Aging and Physical Activity, 4, 25-32.

Dechamps, A., Onifade, C., Decamps, A., \& Bourdel-Marchasson, I. (2009). Health-Related Quality of Life in Frail Institutionalized Elderly: Effects of a Cognition-Action intervention and Tai Chi, Journal of Aging and Physical Activity, 17, 236-248.

Fong, S.M., \& Ng, G.Y. (2006). The Effects on Sensoriomotor Performance and Balance with Tai Chi Training, Archives of Physical Medicine and Rehabilitation, 87(1), 82-87.

Galantino, M.F., Shepard, K., Karft, L., Laperriere, A., Ducette, J., et al. (2005). The Effect of Group Aerobic Exercise and T'ai Chi on Functional Outcomes and Quality of Life for Persons Living 
with Acquired Immunodeficiency Syndrome, The Journal of Alternative and Complementary Medicine, 11(6), 1085-1092.

Gyllensten, A.L., Hui-Chan, C., \& Tsang, W. (2010). Stability Limits, Single-Leg Jump, and Body Awareness in Older Tai Chi Practitioners, Archives of Physical Medicine and Rehabilitation, 91, 215-220.

Hall, A., Maher, C., Latimer, J., \& Ferreira, M. (2009). The Effectiveness of Tai Chi for Chronic Musculoskeletal Pain Conditions: A Systematic Review and Meta-Analysis, Arthritis \& Rheumatism, 6, 717-724.

Hernandez-Reif, M., Field, T.M., \& Thimas, E. (2001). Attention Deficit Hyperactivity Disorder: benefits from Tai Chi. Journal of Bodywork and Movement Therapies, 5(2), 120-123.

Hoffmann-Smith, K.A., Ma, A., Yeh, C.T., Deguire, N.L., \& Smith, J.P. (2009). The Effect of Tai Chi in Reducing Anxiety in an Ambulatory Population, Journal of Complementary and Integrative Medicine, 6(1), 1-12.

Hosseini, H., Fakhari, M., Marandi, S.M., \& Rezaie, A. (2011). The Effect of Tai Chi Exercise on the Sleep Quality of the Elderly residents in Isfaham Sadeghieh Elderly Home, Iran Journal of nursing and midwifery research, 16(1), 55-50.

Irwin, M., Olmstead, R., \& Motivala, S. (2008). Improving Sleep Quality in Older Adults with Moderate Sleep Complaints: A Randomized Controlled Trial of Tai Chi Chih, SLEEP, 31(7), 1001-1008.

Jannke, R.A., Larkey, L.K., \& Rogers, C. (2010). Dissemination and Benefits of a Replicable Tai Chi and Qigong Program for Older Adults, Geriatric Nursing, 31(4), 272-280.

Jiménez, P.J., Meléndez, A. \& Albers, U. (2012). Psychologycal Effects of Tai Chi Chuan, Archives of Gerontology and Geriatrics, DOI 10.106/j.archger.2012.02.003.

Jin, P. (1989). Changes in Heart Rate, Noradrenaline, Cortisol and Mood during Tai Chi. Journal of Psychosomatic Research, 33(2), 197-206.

Jin, P. (1992). Efficacy of Tai Chi, Brisk Walking, Meditation, and Reading in Reducing Mental and Emotional Stress. Journal of Psychosomatic Research, 36(4), 361-370.

Kemper, K., Bulla, S., Krueger, D., Ott, M.J., McCood, J.A., et al. (2011). Nurses' experiences, expectations, and preferences for mind-body practices to reduce stress. BMC Complementary and Alternative Medicine, 11(26), 1-9.

Kozasa, E.H., Hachul, H., Monson, C., Pinto, L., Csermak, M., et al. (2010). Mind-body interventions for threatment of insomnia: a review. Revista Brasileira de Psiquiatría, 32(4), 437-443.

Kuramoto, A. (2006). Therapeutic Benefits of Tai Chi Exercise: Research Review. Wisconsin Medical Journal, 105(7), 42-46.

Lavretsky, H., Alstein, L.L., Olmstead, R.E., Ercoli, L.M., Riparetti-Brown, M., Cyr, N., Irwin, M.R., (2011) Complementary use of Tai Chi Chih Augments Escitalopram Treatment of Geritaric Depression: A Randomized Controlled Trial, American Journal of Geritaric Psychiatry, 19(10), 839-850.

Lee, H.J., Park, H.J., Chae, Y., Kim, S.Y. Kim, S.N., et al. (2009). Tai Chi Qigong for the quality of life of patients with knee osteoarthritis: a pilot, randomized, waiting list controlled trial, Clinical Rehabilitation, 23, 504-511.

Lee, M.S., Pittler, M.H., \& Ernst, E. (2008). Tai Chi for osteoarthritis: a systematic review. Clinical Rheumatology, 27, 211-218.

Li, F., Fisher, J. Harmer, P., Irbe, D., Tearse, R.G., et al. (2004). Tai Chi and Self-Rated Quality of Sleep and Daytime Sleepiness in Older Adults: A Randomized Controlled Trial. J. American Geriatrics Society, 52 (6), 892-900.

Li, F., Harmer, P., Mack, K.A., Sleet, D., Fisher, K.J., et al. (2008). Tai Chi: moving for better balance development of a community - based falls prevention program, Journal of Physical Activity and Health, 5(3), 445-455.

Li, J.Y., Zhang, Y.F., Smith, GS., Xue, C.J., Luo, Y.N., et al. (2011). Quality of Reporting of Randomized Clinical Trials in Tai Chi Interventions - A systematic Review. Evidence-Based Complementary and Alternative Medicine, 1-10.

Low, S., Ang, L., Goh, K., \& Chew, S. (2009). A systematic review of the effectiveness of Tai Chi on fall reduction among the elderly. Archives of Gerontology and Geriatrics, 48, 325-331.

Ni, G.X., Song, L., Yu, B., Huang, C.H., \& Lin, J.H. (2010). Tai Chi Improves Physical Function in Older Chinese Women with Knee Osteoarthritis, Journal of Clinical Rheumatology, 16(2), 64-67. 
Pérez-Gutiérrez, M.; Gutiérrez-García, C., \& Escobar-Molina, R. (2011). Terminological recommendations for improving the visibility of scientific literature on martial arts and combat sports, Archives of Budo, 7(3), 159-166.

Rogers, C., Keller, C., \& Larkey, L. (2010). Perceived Benefits ot Meditative Movement in Older Adults, Geriatric Nursing, 31, 37-51

Rogers, C., Larkey, L., \& Keller, C. (2009). A Review of Clinical Trials of Tai Chi and Qigong in Older Adults, West Journal of Nursing Research, 31(2), 245-279.

Sarris, J., \& Byrne, G. (2011). A systematic review of insomnia and complementary medicine. Sleep Medicine Reviews, 15 (2), 99-106.

Song, R., Lee, E.O., Lam, P., \& Bae, S.C. (2003). Effects of tai chi exercise on pain, balance, muscle strength, and perceived difficulties in physical functioning in older women with osteoarthritis: a randomized clinical trial, Journal of Rheumatology, 30(9), 2039-2044.

Sood, A., Narayanan, S., Wahner, D.L., Knudsen, K., Sood, R., et al. (2007). Use of Complementary and Alternative Medicine Treatments by Patients with Obstructive Sleep Apnea Hypopnea Syndrome, Journal of Clinical Sleep Medicine, 3(6), 575-579.

Spirduso, W., Francis, K., \& Macrae, P. (2005). Physical Dimensions of Aging. United States: Human Kinetics.

Taboonpong, S., Putshsri, N., Kong-In, W., \& Saejew, A. (2008). The Effects of Tai Chi on Sleep Quality, Well Being and Physical Performance among Older Adults, Thai Journal of Nursing Research, 12, 1-13.

Taggart, H. (2002). Effects of Tai Chi Exercise on Balance, Functional Mobility, and Fear of Falling Among Older Women, Applied Nursing Research, 15(4), 235-242.

Taylor-Piliae, R.E., \& Froelicher, E. (2004). Measurement properties of Tai Chi exercise self-efficacy among ethnic Chinese with coronary heart disease risk factors: a pilot study. European Journal of Cardiovascular Nursing, 3, 287-294.

Taylor-Piliae, R.E., Haskell, W.L., Waters, C., \& Froelicher, E.S. (2006). Change in perceived psychosocial status following a 12-week Tai Chi exercise programme. Journal of Advanced Nursing, 54(3), 313-329.

Toda, M., Den, R., Hasegawa-Ohira, M., \& Moritomo, K. (2011). Influence of personal patterns of behaviour on the effects of Tai Chi: a pilot study. Environmental Health and Preventive Medicine, 16, 61-63.

Tsang, W.W., Wong, V.S., Fu, S.N., \& Hui-Chan, C.W. (2004). Tai Chi Improves Standing Balance Control Under Reduced or Conflicting Sensory Conditions. Archives of Physical Medicine and Rehabilitation, 85, 129-137.

Valenza, M.C., Rodenstein, D.O., \& Fernández, C. (2010). Consideration of sleep dysfunction in rehabilitation, Journal of Bodywork \& Movement Therapies, 15, 262-267.

Wall R. (2005). Tai chi and mindfulness-based stress reduction in a Boston Public middle School. Journal of Pediatrics and Health Care, 19(4), 230-237.

Wang, C., Collet, J.P., \& Lau, J. (2004). The Effect of Tai Chi on Health Outcomes in Patients With Chronic Conditions. Archives of Internal Medicine. 164, 493-501.

Wang, C., Schmid, C.H., Rones, R., Kalish, R., Yinh, J., Goldenberg, D.L., Lee, Y., McAlindon, T. (2010). A randomized trial of tai chi for fibromyalgia. New England Journal of Medicine, 363 (8), 743754.

Wang, C., Raveendhara, B., Ramel, J. Kupelnick, B., Scott, T., et al. (2010a). Tai Chi on psychological well-being: systematic review and meta-analysis, BMC: Complementary, \& Alternative Medicine, 10(23), 1-16.

Wang, W.C. Zhang, A.L., Rasmussen, B., Lin, L.W., et al. (2009). The Effect of Tai Chi on Psychological Well-Being: A systematic review of randomized controlled trials. Journal of Acupuncture and Meridian Studies, 2(3), 171-181

Wolf, S.; Barnhart, H.; Kutner, N.; McNeely, E.; Coogler, C., et al. (1996). Reducing Frailty and Falls in Olders Persons: An Investigation of Tai Chi and Computerized Balancing Training, Journal of the American Geriatrics Society, 44(5), 489-497.

Wong, A.M., Lin, Y.C., Chou, S.W., Tang, F.T., \& Wong, P.I. (2001). Coordination Exercise and Postural Stability in Elderly People: Effect of Tai Chi Chuan, Archives of Physical Medicine and Rehabilitation, 82, 608-612. 
Yau, M., \& Packer, T. (2002). Health and well-being through T'ai Chi: perceptions of older adults in Hong Kong. Leisure Studies, 21, 163-178.

Yeh, G., Wang, C., Wayne, P., \& Phillips, R. (2009). Tai Chi Exercise for Patients with Cardiovascular Conditions and Risk Factors: A Systematic Review. Journal of Cardiopulmonary Rehabilitation and Prevention, 29(3), 152-160.

Yeh, G., Mietus, J., Peng, C.K., Phillips, R., Davis, R., et al. (2008). Enhancement of sleep stability with Tai Chi exercise in chronic heart failure: Preliminary findings using an ECG-based spectrogram method, Sleep Medicine, 9, 527-536.

Zhang, L., Layne, C., Lowder, T., \& Liu, J. (2011). A review focused on the Psychological Effectiveness of Tai Chi on Different Populations, Evidence-Based Complementary and Alternative Medicine, $1-9$.

Zhang, J.G., Takata, K.I., Yamazaki, H., Morita, T., \& Ohta, T. (2006). The effects of Tai Chi Chuan on physiological function and fear of falling in the less robust elderly: An intervention study for preventing falls. Archives of Gerontology and Geriatrics. 42(2), 107-116.

\section{Datos biográficos de los autores}

Pedro Jesús Jiménez es Doctor en Ciencias de la Actividad Física y del Deporte y profesor de la asignatura Tai Chi Chuan en la Facultad de Ciencias de la Actividad Física y del Deporte-INEF de la Universidad Politécnica de Madrid. Se inició en la práctica del Tai Chi Chuan estilo Yang en el año 1993; fue practicante de TCC estilo Chen en el Círculo del Retiro con el profesor Liang Puwan (2004-2009), y del estilo Yang con el profesor Haoqing Liu en la Escuela Superior de Chikung-Taichi Haoqing Liu y Medicina Tradicinal China (2008-2011). Director de diferentes cursos de TCC y Chi Kung en la FCAFYD-INEF de la UPM (2007-2011). 1oㅡ Dan de Kendo y 1ํㅡㅁㅡ De Iaido. E-mail: pedrojesus.jimenez@upm.es. 